\title{
Strapdown inertial navigation systems readings correction based on navigational data of other sensors and systems with intelligent selection of the priority adjuster
}

\author{
P.M. Trefilov ${ }^{1, *}, M . V$. Mamchenko $^{1}$, and A.V. Korol'kov ${ }^{2}$ \\ ${ }^{1}$ Institute of Control Sciences of Russian Academy of Sciences, 65, Profsoyuznaya str., 117997, \\ Moscow, Russia \\ ${ }^{2}$ Russian Technological University (MIREA), 78, Vernadsky ave., 119454, Moscow, Russia
}

\begin{abstract}
Strapdown inertial navigation systems (SINS) are one of the main components of the navigation systems of the drones and aircraft (including autonomous ones), but their readings need to be instanly corrected due to the constant accumulation of errors. This paper comprises the review of existing approaches to using one or more sensors or systems to correct the navigation data of SINS algorithms (herein after - correctors) using integrated information processing. A common disadvantage of the analysed approaches is the lack of flexibility concerning the types and the number of SINS correctors used, as well as the growth of computational burden due to the use of the measurement vectors of all the correctors in the process of forming the state vector of the system. This article proposes the use of the original adaptive scheme based on the selection of the least noisy data, taking into account environmental conditions, for the integrated processing of the SINS and the correctos' navigation parameters. The essence of the approach is that the state vector is estimated on the basis of the most reliable corrector. This allows reducing the correlation of errors in the correctors' measurement of navigational parameters, since only the measurement vectors (or vector) with best navigational data signal/noise ratio (received from the corresponding correctors) are used in forming the state vector. Furthermore, the proposed navigational data fusion scheme has a modular structure and greater flexibility in comparison with the loosely coupled systems, and also implies the use of an arbitrary number of correction sensors and systems regardless of the physical nature of their measurements.
\end{abstract}

\section{Introduction}

SINS are currently one of the main elements of aviation navigation systems. Compared to the platform inertial navigation systems, SINS are advantageous, inter alia, due to the

\footnotetext{
* Corresponding author: petertrfi@gmail.com
} 
increased informativeness of output navigation data, reliability and resistance to mechanical impacts, reduced mass and energy consumption.

However, due to the constant accumulation of errors due to the SINS own errors, it is needed to be corrected [1]. Therefore, it is not currently possible to use SINS exclusively for the purposes of autonomous navigation of unmanned aerial vehicles (UAV) and aircraft.

Approaches to the improvement of navigation efficiency using SINS can be grouped broadly in the following two areas:

1. Improvement of the SINS architecture and its algorithms. This set of approaches includes the development and implementation of fundamentally innovative inertial measurement units (e.g., fibre-optic gyroscopes (FOG), triads of micromechanical gyroscopes and accelerometers (MEMS - Micro-Electro Mechanical Systems) [2]), improvement of the SINS configiration (e.g., introducing the reserving measurement channels through the duplication of measuring instruments - the integration of distributed SINS based on FOG and MEMS into a tightly coupled system [3]), as well as new algorithms and methods of filtering and estimating the output navigation parameters (Kalman filter varieties: extended, cubature, optimal, and sigma-point [4]; Wiener filter [5]; minimax, and adaptive restrictive filters [6]; wavelet transforms [7]; the use of artificial neural networks [8], etc.).

2. Adjustment of the SINS errors through the integrated processing of navigation data using other navigation information sources and measurement systems.

The aim of this work is to analyse the existing schemes for the integration of navigational data and the correction of SINS readings with the aid of other means for the provision of navigational information, as well as to describe the proposed approach to the integrated fusion and processing of the navigational information. Due to the impossibility of considering all the issues of improving the efficiency of SINS-based navigation systems, this paper will deal exclusively with the navigation data fusion schemes using other sources of navigation data. The problem of SINS initial alignment is not considered. In addition, the next section will deal exclusively with navigational sensors and systems used to correct (adjust) SINS readings, which are entirely a part of the on-board avionics of the UAVs or the aircraft.

\section{Approaches to SINS readings and other navigation data fusion schemes}

Let's consider the main options for the forming the state vector and difference of measurements when fusing the navigation data from different on-board sensors and systems in order to correct the SINS readings:

1. Separate (non-coupled) system. The basic principle of the scheme is the independent work of the SINS and the adjuster (usuall - the Global Navigation Satellite System (GNSS) receiver) from each other with periodic restarts of the SINS operation algorithm with simultaneous correction of the coordinates and velocity using the readings of the corrector. This scheme is characterised by the independence of the two navigation subsystems, information redundancy, minimal changes in the composition of the on-board equipment [6].

2. Loosely coupled system. SINS and the corrector in the loosely coupled system work functionally (sometimes - even physically) in a separate way from each other (the resulting output of the two navigation systems are essentially independent), but the SINS data is corrected algorithmically in an independent computational and correction unit (most often by forming the state vectors estimation using the Kalman filter). Compared to the separate system, this scheme allows to perform SINS initial alignment and calibration during the flight $[4,6]$. 
3. Tightly coupled system. SINS defines the primary parameters of the forward and rotational motions. Measurements from the SINS and the corrector are transmitted to a single calculation unit with the Kalman filter. Compared to a loosely coupled system, the tightly coupled navigation system has better overall interference resistance, but less redundancy due to only one Kalman filter [6].

4. Deeply integrated system. SINS errors, pseudo range and pseudo velocity estimates are computed in a single calculation unit with an integrated Kalman filter, which leads to futher simplification of the GNSS unit [9]. An additional parallel Kalman filter is introduced for the GNSS receiver to eliminate the lack of information redundancy compared to tightly coupled schemes. This scheme also ensures the best positioning accuracy of the UAV/aircraft, but is computing resources intensive and requires sufficient reconfiguration of the navigation avionics [6].

\section{Sensors and systems used for the sins readings correction}

\subsection{Global navigation satellite systems}

The use of GNSS to correct SINS data is an implicit standard in the current navigation data fusion schemes [10]. Not only GPS, but also GLONASS and BeiDou receivers can be used [11]. In general, these type of correction systems can be characterized as follows: SINS navigation data with a time-dependent decreasing precision and a high update rate are corrected by satellite data with a high location accuracy and a low update rate [12]. Thus, it allows taking the advantages of each of the navigation subsystems [4]. The main drawback is the sensitivity of the GNSS unit to the electromagnetic interferences [13], which may result in the inaccessibility of satellite navigation data and will force the SINS to switch to a standalone uncorrected operation mode.

Specific studies of such systems are as follows:

- SINS readings correction via GNSS data in a tightly coupled scheme with the use of the Cubature Kalman filter [14];

- dual-channel navigation system with the scheme of dual-channel fusion of SINS and GNSS data [15];

- the use of GNSS data to adjust the FOG-based SINS [16];

- miniature inertial measurement unit (MIMU, a set of MEMS gyroscopes and accelerometers)/GPS navigation data fusion schemes [17, 18].

The SINS/GNSS data fusion schemes have long been considered optimal. Despite this, currently other navigation sensors and systems (designed to be used as SINS data correctors) are being developed because of the risks of possible inaccessibility of satellite data.

\subsection{Correlation-extreme navigation systems}

The working principle of the correlation-extreme navigation systems (CENS) is the comparison of «images» of the Earth physical fields (EPF), where the current «image» is compared with the previously received one (or other reference «image»). Differences in two images in the given coordinate system allow to form the trajectory of motion of the dynamic object [19]. EPF comprise the spatial and the surface fields.

Spatial fields include geomagnetic and gravitational fields. Geomagnetic CENS (magnetometric navigation systems) may be magnetogradient or magnetic field anomalybased [20]; these navigations sustems require accurate digital maps of the Earth's geomagnetic fields [21], and high-precision magnetic field sensors on board the aircraft/UAV [22]. Kalman filter (i.e. the Extended Kalman Filter) and the Marginalized Particle Filter can 
be used as the algorithms within these systems [23]. The main principle of the gravitational CENS is the use of gravity meters and gravy-gradient meters to measure the anomalies of the Earth's gravitational field [24].

Surface CENS obtain navigation data via the terrain, radiothermal and radar fields. Surface-based CENS (or visual-aided navigation systems - VANS) can use both satellite images and aerial survey images as the reference ones [13]. The working principle of such CENS can also be based on photogrammetric processing of the images. There are a number of approaches for the surface CENS to obtain navigation data, including:

- image processing based on known coordinates of artificial or natural surface reference points $[25,26]$

- the use of the optoelectronic systems (OES) combined with a set of digital maps (images) of the surface (SDM - surface digital maps; two-dimensional images or maps) or orthophotoplans [2];

- the use of the OES combined with the three-dimensional digital model of the surface $[27,28])$.

The radiothermal field-based CENS use the gyrostabilized thermal imaging sensors and allow to determine the azimuth to the objects with known coordinates and their distance from the aircraft/UAV [29].

Synthetic aperture radars are most commonly used in radar field-based CENS, obtaining the navigational data via the comparison of time-separated periodic measurements of the radar «situation» (radar-based CENS may also use a priori radar filed digital map: i.e., in the study [30] such a system uses the Markov chain Monte Carlo method, the regularized particle filter algorithm and the extended Kalman filter, while the paper [31] describes the use of the Random Weighting Filtering algorithm).

In general, the use of different types of CENS as the correctors of the SINS readings increases the overall information redundancy of the aircraft/UAV navigation system, including when GNSS signals are not avialable [25].

\subsection{Air data computer}

Air data computer (or air data system) provides information on the altitude and velocity parameters of the aircraft/UAV to correct the navigation readings of the SINS (the example of such navigation data fusion system is shown in [32]).

\subsection{Dead reckoning using dynamic recurrent correction systems}

Dynamic recurrent correction is a method for determining the position of the moving object in space when the navigational parameters are constrained by the dynamic properties of the moving object itself. Thus, the aircraft/UAV dynamics model is formed [33]. When combined with the Dead Reckoning method data, the aircraft's/UAV's current position is calculated taking into account the dynamic model of the object, its previous position, velocity, course, and time difference between the two measurements [34]. Navigational data fusion schemes of SINS and dynamic recurrent correction systems readings are presented in $[33,35]$.

\subsection{Celestial navigation system}

Celestial navigation systems (CNS) imply the fusion of SINS readings and navigation data from the star-tracker [36]. These systems are desinged to be primarily installed on ballistic missiles and space objects. Star-tracker are also rarely used on board the high-altitude 
aircraft, and their deployment on drones makes no sense at all. Despite this, SINS/CNS navigation data fusion studies are still being conducted (i.e., [37]).

\subsection{Doppler velocity and drift angle measuring device as a special case of doppler navigation}

The Doppler Radar Navigation Method is based on the Doppler Effect and involves the use of the Doppler Velocity and Drift Angle Measuring Device (DVDAMD) - a multi-channel radar with a narrow beam pattern for continuous measurements of the speed vector, the drift angle, current position coordinates of the aircraft/UAV [38]. As an example, it is worth mentioning the study [39], describing a fusion scheme of FOG-based SINS and the Doppler radar navigation data with the use of the Kalman filter. DVDAMD is capable of providing navigation data when flying over any type of surface, and has a beyond line-of-sight capability.

In general, Doppler navigation is now actively used in helicopter avionics.

\subsection{Combined navigation systems and multiple sensor correction systems}

The most advanced approach to the fusion of navigational data is processing navigation readings from multiple correctors simultaneously. There are studies and solutions that use multiple sensors in different fusion schemes to adjust SINS data. Such approaches can further increase the information redundancy and reduce errors of the aircraft's/UAV's positioning.

The standard approaches are to complement the SINS/GNSS navigation data fusion system with additional sensors; but there are also other approaches where the GNSS receivers are not used at all. The existing studies and examples of the use of different sets of sensors and systems for navigational data fusion and correction of SINS readings are as follows:

SINS/GNSS/barometric altimeter (for the purposes of SINS vertical channel damping) in the separate, loosely, and tightly coupled fusion schemes [40];

SINS/GNSS with a differential global positioning system/barometric altimeter [41];

SINS/GNSS/magnetic compass [4];

SINS/GNSS/dynamic recurrent correction system [35];

SINS/GNSS/VANS [12];

SINS/GNSS/Doppler radar [34];

FOG-based SINS/Doppler radar/barometric altimeter [39];

SINS/GNSS (three receivers)/remote magnetometer within the magnetometric CENS/Air Data Computer/radio altimeter/differential global positioning system [42];

SINS/GNSS/magnetic compass/Air Data Computer/radio altimeter/OES- and SDMbased CENS [43];

FOG- or MEMS-based SINS/GNSS/barometric altimeter/triaxial magnetometer [2].

\subsection{A shift towards integrated modular navigation avionics}

The most promising approaches are the development of a reconfigurable modular integrated navigation system, that would be able to incorporate heterogeneous sensors operating on different physical principles, so that the number of the used sensor and the composition of such a navigation system could be easily reconfigured. Such an architecture would meet modern requirements and trends in the field of creation and implementation of integrated modular avionics. So, both the navigation systems and its elements should be modular and standardized. 
A similar system was described as a concept of the on-board navigation module in the articles [32, 43]. However, in the above-mentioned concepts of an integrated modular navigation system, the estimation errors resulting from the measurement errors of the navigational parameters will correlate with each other, since the general state vector is formed from all measurement vectors of the SINS and the correctors at once.

It is therefore necessary to reduce the correlation of errors in the corrector's navigational measurements within these integrated modular navigation systems. The authors propose to use in the process of forming the state vector only those measurement vectors with the highest signal/noise ratio of the corresponding navigational data obtained from the corresponding correctors.

\section{The proposed navigation data fusion scheme}

The measurement vector is formed on the basis of data received from SINS, GNSS and an arbitrary number of heterogeneous correctors. Navigational data are transmitted to high-pass and low-pass filters to remove rough errors. The measurement vector is formed in a similar way to the loosely coupled navigational data fusion scheme - the difference of the measurements of the SINS/correctors is formed. This expression is shown in the formula (1):

$$
\begin{aligned}
& \overline{Z_{1}}=\bar{Z}_{\text {SINS/GNSS }}+\bar{J} \\
& \quad \overline{Z_{2}}=\bar{Z}_{\text {SINS/Cor } 1}+\bar{J} \\
& \overline{Z_{n}}=\bar{Z}_{\text {SINS/Corn }}+\bar{J}
\end{aligned}
$$

where $\bar{J}$ is the vector representation of the environmental parameters.

The analytical module then compares the signal/noise ratios of the measurement vectors relative to each other and supplements them with data from external environmental sensors (temperature, humidity, illumination, etc.) [44]. The least noisy measurement vector (or group of least noisy measurement vectors) are then selected; these vectors (or this vector) will be a basis to form the general state vector of the dynamic system $\bar{X}$ :

$$
\bar{X}=
$$

where $x_{1}, x_{2}, x_{3}$ - SINS errors of the horizontal coordinates and the altitudes computation; $x_{4}, x_{5}, x_{6}-$ SINS errors of the calculation of the horizontal and the vertical velocity projections; $\propto, \beta, \gamma-$ small angular rotation error of the true horizon due to its imperfect calculation by the SINS (relative to its ideal position); $\Delta \bar{\Omega}_{x, y, z}, \Delta \bar{n}_{x, y, z}-$ instrumental errors of the gyroscopes and accelerometers.

The correction values of SINS and correction devices are formed in the Optimal Kalman Filter unit on the basis of this vector (Fig. 1).

The distinctive feature of the proposed approach compared to the traditional loosely coupled scheme of navigational data fusion with the use of multiple sensors (i.e., in [32, 43]) is the reduction of the mutual correlation of errors accumulated by the adjusters with different physical principles of measurements. In addition, environmental data are also taken into account, which increases the reliability of the received navigational information and allows assessing the performance of the corrector under various external influences. 


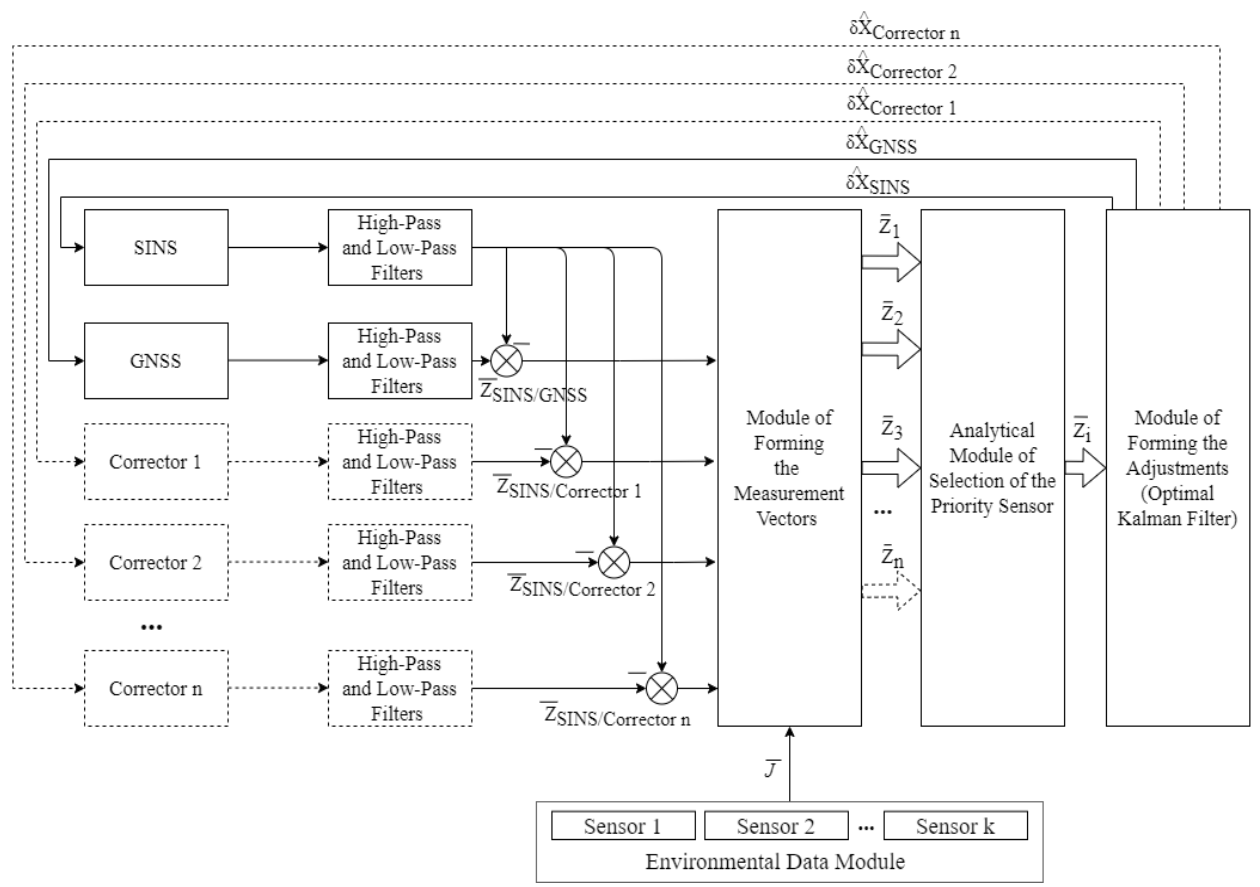

Fig. 1. SINS/correctors navigation data fusion scheme with forming a set of measurement vectors.

\section{Conclusion and further work}

The autonomous work of the strapdown inertial navigation systems is limited and its readings should be adjusted. In this paper the authors analyzed the existing schemes of navigation data fusion schemes, and approaches to integrated processing of navigation information from the measuring devices, as well as methods of correction of the SINS algorithms of work. All analyzed approaches allow to reduce the accumulation of the SINS errors.

The analyzed SINS readings correction schemes have their advantages and disadvantages, but all of them do not take into account the influence of the factors of external environment on the operation of the correctors. The authors propose an approach to the fusion of navigation data and their integrated processing based on the loosely coupled scheme, that considers the environmental conditions. In particular, the proposed approach implies the vector of measurements to be extended by the environmental data. Comparison of the signalto-noise ratio of the navigation data of the corresponding adjusters allows to form a single state vector of the dynamic system from the least noisy measurement vectors. Such a scheme could have great potential in the field of automatic control of the aircraft and the drones, and should improve the accuracy of obtained navigational parameters under unfavorable environmental conditions.

Further computer simulations, modeling, and related field experiments are planned.

\section{References}

1. Trefilov P M 2019 Proceedings of the 13th All-russian Meeting on Management Problems (WSPU 2019) p 470

2. Sizov A V, Ippolitov S V, Savchenko A Yu and Malishev V A 2018 Modelling, 
optimization and information technology 6(4) 381

3. Chernodarov A V, Patrikeev A P, Merkulova I I and Ivanov S A 2017 Civil Aviation High Technologies 20(6) 111

4. Al Bitar N and Gavrilov A I 2019 Giroskopiya i Navigatsiya 27-3(106) 31

5. Raspopov V Y, Ivanov Y V, Alaluev R V, Shukalov A V, Pogorelov M G and Shvedov A P 2013 Autom Remote Control 742189

6. Uljanov G N and Kolesnichenko S V 2015 Defence equipment issues. Series 16: Counter-Terrorism Technical Means 11-12 (89-90) 29

7. Amosov O S and Amosova S G 2016 Information technology and management systems 1 (47) 82-94

8. Song R and Chen X 2017 IEEE International Workshop on Metrology for AeroSpace (MetroAeroSpace) p 201

9. Suvorov M A 2015 Current science and education challenges 1-1 408

10. Fomichev A V and Tan' L 2015 Science and Education of the Bauman MSTU 10252

11. Kang X, He G and Li X 2019 Mathematical Problems in Engineering 2019 1-6

12. Duo J and Zhao L 2017 Proceedings of the 36th Chinese Control Conference p 5777

13. Artyshenko A and Kamaev M 2014 IEEE 3rd International Conference on Methods and Systems of Navigation and Motion Control (MSNMC) Proceedings $\mathrm{p} 112$

14. Gao M, Zhao X, Li Q and Wang Zh 2017 13th International Conference on Natural Computation, Fuzzy Systems and Knowledge Discovery (ICNC-FSKD 2017) p 1077

15. Norseev S A, Burov D A and Tyutyugin D Yu 2020 Izvestiya TulGU. Technical Sciences 13

16. Wang H, Wang M, Wen K and Wu W 2017 Proceedings of the 36th Chinese Control Conference p 6157

17. Zhang F, Shan B, Wang Yu, Hu Y, Guo Zh and Teng H 2018 IEEE CSAA Guidance, Navigation and Control Conference (CGNCC) 1

18. Zhang X and Song Ch 2013 Applied Mechanics and Materials 278-280 1719

19. Dzhandzhgava G I and Sazonova T V 2016 Izvestiya SFedU. Engineering Sciences p 102

20. Karshakov E 2011 Large system management 35265

21. Minligareev V T, Alekseeva A V, Kachanovsky Y M, Repin A Y and Khotenko E N 2019 Izvestiya SFedU. Engineering Sciences 1(203) 248

22. Tkhorenko M Yu, Pavlov B V, Karshakov E V and Volkovitsky A K 2018 25th Saint Petersburg International Conference on Integrated Navigation Systems (ICINS) p 1

23. Xie F, Dong M Zh, Zhang H D and Ru K 2019 International Conference on Unmanned Systems and Artificial Intelligence (ICUSAI) p 19

24. Affleck C A and Jircitano A 1990 IEEE Symposium on Position Location and Navigation. A Decade of Excellence in the Navigation Sciences $\mathrm{p} 60$

25. Antonov D A, Zharkov M V, Kuznetsov I M, Lunev E M and Pron'kin A N 2016 MAI Proceedings 911

26. Dong Zh, Li W and Zhou Ya 2016 IEEE Chinese Guidance, Navigation and Control Conference p 982

27. Bondarev V G 2015 MGTU GA Scientific Bulletin 21365

28. Kovalenko A M and Sheinikov A A 2020 System analysis and applied information 
science 217

29. Shakhrai V I and Tazuk D G 2013 Communications Technology. Series: Television Technology 144

30. Gao Yi, Gao Sh and Gu Yu 2010 International Conference on Computer Application and System Modeling (ICCASM 2010) V9 70

31. Yu G, Mi M and Jian-hua G 2010 IEEE 10th International Conference On Signal Processing Proceedings p 2531

32. Savel'ev V M and Antonov D A 2011 MAI Proceedings 452

33. Jieliang S, Xinhua Z, Yu W and Yan S 2016 DGON Intertial Sensors and Systems (ISS) p 1

34. Zhenguo Y 2019 6th International Conference on Information Science and Control Engineering (ICISCE) p 1068

35. Telny A V and Yakovleva E I 2019 Promising technologies in the means of transferring information $\mathrm{p} 78$

36. Korovin A V, Soloviev Yu A and Ustiuzhanin D A 2012 Methodological issues of estimating an expected astroinertial satellite system accuracy Navigation News 1 31-23

37. Xiaolin N, Weiping Y and Yanhong L 2019 Journal of Systems Engineering and Electronics 30(4) 770

38. Lomanov A N and Revunov V V 2016 Bulletin of the P. A. Solovyov Rybinsk State Aviation Technology Academy 1(36) 108

39. Bai H and Xue X 2010 Second International Conference on Computer Modeling and Simulation $\mathrm{p} 115$

40. Ragimov E A Andrievskaya V Yu and Alexeev A V 2015 Proceeding of the Military space academy named after A.F. Mozhaiskiy 64663

41. Shatokhin M V and Nikitin O R 2008 Methods and devices for transmitting and processing information 10182

42. Veremeenko K K, Antonov D A, Zharkov M V, Zimin R Yu, Kuznetsov I M and Pron'kin A N 2011 Navigation News 422

43. Aleshin B S, Antonov D A, Veremeenko K K, Zimin R Yu, Zharkov M V, Kuznetsov I M and Pron'kin A N 2012 MAI Proceedings (Trudi MAI) 541

44. Mamchenko M V, Romanova M A, Trefilov P M 2020 International Conference on Industrial Engineering, Applications and Manufacturing (ICIEAM) p 1 